\title{
Dental anomalies as a possible clue of 1 p36 deletion syndrome due to germline mosaicism: a case report
}

\author{
D. Nistico ${ }^{\prime 1^{*}}$, F. Guidolin ${ }^{2}$, C. O. Navarra ${ }^{3}$, M. Bobbo $^{3}$, A. Magnolato $^{3}$, A. P. D'Adamo ${ }^{1,3}$, E. Giorgio ${ }^{4}$, B. Pivetta ${ }^{5}$, \\ E. Barbi ${ }^{1,3}$, P. Gasparini ${ }^{1,3}$, M. Cadenaro ${ }^{1,3}$ and F. Sirchia ${ }^{3}$
}

\begin{abstract}
Background: Monosomy 1 p36 is the most common terminal deletion syndrome with an autosomal dominant pattern of inheritance. This syndrome is defined by an extremely wide spectrum of characteristics; however, developmental delay and intellectual disability of various degree are present in all patients and about the 90\% of patients have a severe intellectual disability. Dental agenesis or other dental anomalies have not been described in previous reports.

Case presentation: We report the case of two little sisters born from healthy and non-consanguineous parents, presenting with dental anomalies and one of them with epilepsy, dilated cardiomyopathy with left-ventricular noncompaction, strabismus, history of poor growth, hypotonia and mild language delay. Patients were evaluated in several departments (genetic, child neuropsychiatric, cardiology, odontostomatology, ophthalmology, otorhinolaryngology) of Institute for Maternal and Child Health, IRCCS Burlo Garofolo, Trieste, Italy. They underwent investigations such as electrocardiogram, echocardiogram, dental orthopantomography X-Ray and Computed Tomography, electroencephalograms, abdomen ultrasound, blood tests, IQ tests, genetic analysis. They both have an Intelligence Quotient greater than 70 and a negative neurologic exam. Each sister carries the same 1 p36 deletion of about 2.3 Mb. Genetic analysis of the parents' blood samples (Single Nucleotide Polymorphism- array, karyotype and Fluorescent In Situ Hybridization) did not reveal any deletion, translocation or inversion and confirmed the paternity. A third sib of the probands does not carry the $1 \mathrm{p} 36$ deletion or other quantitative alterations.
\end{abstract}

Conclusion: This report describes a new trait linked to monosomy 1 p36, namely a mild intellectual outcome associated with significant dental anomalies. Our finding suggests that 1 p36 deletion syndrome may present with a mild cognitive impairment or even with a normal intellectual development: this is very important for the genetic counselling, especially in a prenatal setting. Moreover, we report the third study with recurrent 1 p36 deletion syndrome in two siblings, likely due to germline mosaicism. Finally, we believe that the dental anomalies should be investigated in 1 p36 deletion syndrome and that the spectrum of the condition could be broader than we assume.

Keywords: 1 p36 deletion syndrome, Monosomy 1p36 syndrome, Germline mosaicism, Dental anomalies, Recurrent microdeletion, Intellectual disability, Case report

\footnotetext{
* Correspondence: daniela.nistico@burlo.trieste.it

${ }^{1}$ University of Trieste, Trieste, Italy

Full list of author information is available at the end of the article
}

(c) The Author(s). 2020 Open Access This article is licensed under a Creative Commons Attribution 4.0 International License, which permits use, sharing, adaptation, distribution and reproduction in any medium or format, as long as you give appropriate credit to the original author(s) and the source, provide a link to the Creative Commons licence, and indicate if changes were made. The images or other third party material in this article are included in the article's Creative Commons licence, unless indicated otherwise in a credit line to the material. If material is not included in the article's Creative Commons licence and your intended use is not permitted by statutory regulation or exceeds the permitted use, you will need to obtain permission directly from the copyright holder. To view a copy of this licence, visit http://creativecommons.org/licenses/by/4.0/ The Creative Commons Public Domain Dedication waiver (http://creativecommons.org/publicdomain/zero/1.0/) applies to the data made available in this article, unless otherwise stated in a credit line to the data. 


\section{Background}

Monosomy $1 \mathrm{p} 36$ is the most common terminal chromosomal deletion in humans [1] and one of the most common microdeletion syndromes [2], with a prevalence of 1:50001:10,000 [3]. Though characterized by autosomal dominant transmission, $52 \%$ of the cases are de-novo terminal deletions, $29 \%$ are de-novo interstitial deletions, $12 \%$ are complex chromosome rearrangements (e.g. more than one 1 p36 deletion or a 1p36 deletion with a 1p36 duplication), $7 \%$ are derivative chromosome 1 (e.g. 1 p telomeric region is replaced by another chromosome end) [4]. Two examples of 1 p36 deletion syndrome in siblings due to germline mosaicism have been described in literature $[2,5]$.

A precise genotype-phenotype correlation and the determination of the genes responsible for specific phenotypic features are not still certainty established [6]. 1p36 deletion syndrome is characterized by an extremely wide spectrum of features including intellectual disability, hypotonia, structural brain abnormalities including white matter anomalies (e.g. periventricular leukomalacia, hypoxic ischemic encephalopathy-like phenotype) [7], epilepsy, behavior disorder, hearing loss, ophthalmologic abnormalities, short stature, congenital heart defects (Ebstein's anomaly, valvular anomalies, tetralogy of Fallot, ventricular septal defects), cardiomyopathy, genitourinary malformations, skeletal anomalies and variable dysmorphisms $[1-3,5,8]$. Developmental delay and intellectual disability of various degree are present in all patients and are hallmarks of the syndrome. Only $10 \%$ of patients with 1 p36 deletion show mild to moderate cognitive impairment, while the remaining $90 \%$ have severe intellectual disability [2]. 1p36 deletion syndrome represents $0.5-1.2 \%$ of total cases of syndromic intellectual disability and it requests multidisciplinary management and intensive follow-up [6] .

Other rare abnormalities associated with 1p36 deletion syndrome include telangiectatic skin lesions and hyperpigmented macules, polydactyly, congenital spinal stenosis, congenital fiber type disproportion myopathy, redundant skin on the nape of the neck, intestinal malrotation, annular pancreas and anomalous arrangement of the pancreaticobiliary duct, liver steatosis, hypertrophic pyloric stenosis, anteriorly placed or imperforate anus, hooked or bilobed gallbladder and small spleen, neuroblastoma (in 3 individuals), pemphigus vulgaris (in 1 individual) [4], cutis laxa [9], biliary atresia [10]. Dental agenesis or other dental anomalies have not been described in previous reports.

\section{Case presentation}

We report on two sisters (6 and 8 years of age) with 1 p36 deletion due to a germline mosaicism in one of the parents, presenting with a new phenotypic trait, namely a mild intellectual outcome associated with significant dental anomalies.

The two sisters described in this report are the only daughters of unrelated parents coming from China.

Both the parents are in good health and no cases of intellectual disability, miscarriage or syndromes have been reported in their families. The patients were evaluated in several departments (genetic, child neuropsychiatric, cardiology, odontostomatology, ophthalmology, otorhinolaryngology) of Institute for Maternal and Child Health, IRCCS Burlo Garofolo, Trieste, Italy. They underwent investigations such as electrocardiogram, echocardiogram, dental orthopantomography X-Ray (OPT-XR) and Computed Tomography (CT), electroencephalograms, abdomen ultrasound, blood tests, Intelligence Quotient (IQ) tests, genetic analysis.

Patient 1 was referred at the age of six for genetic counselling because of suspected learning disorders according to the Diagnostic and Statistical Manual of Mental Disorders, Fifth Edition (DSM-V) [11]. She was born at the $40 \mathrm{GW}$ via spontaneous vaginal delivery following an uncomplicated pregnancy. Ultrasound monitoring was normal and invasive prenatal analysis had not been performed. Birth weight was $3030 \mathrm{~g}$ (50-90th centile) [12], birth length was $48 \mathrm{~cm}$ (10-50th centile) [12] and birth Occipito Frontal Circumference (OFC) was 34 cm (50-90th centile) [12]. Her neonatal history was unremarkable, including negative hearing screening. She was breastfed for five months and then regularly weaned. Developmental milestones were normal. She sat-up at 6 months of age, walked at 13 months and spoke at 9 months. No history of seizures, fractures or dislocations was reported. Genetic analysis (Single Nucleotide Polymorphism (SNP)-array) identified a deletion 1p36.32 of about $2.3 \mathrm{Mb}$, typical of the $1 \mathrm{p} 36$ deletion syndrome. The patient was clinically evaluated with abdomen ultrasound, blood tests, cardiologic consult with electrocardiogram and echocardiogram that were all negative. Intellectual developmental evaluation at 6-year-old with the Wechsler Preschool and Primary Scale of Intelligence, Third Edition (WPPSI-III) showed a total IQ of 82. Now she attends the primary school full time without any support. She speaks Italian and Chinese and she studies English at school. She was recently evaluated again with the Wechsler Intelligence Scale for Children, Fourth Edition (WISCIV) reporting an IQ of 75. At the time of the last visit (8 years), her height was $119.8 \mathrm{~cm}\left(3-10^{\circ}\right.$ centile), her weight was $20 \mathrm{~kg}\left(<3^{\circ}\right.$ centile). Physical examination was remarkable for a pronounced ligamentous laxity of elbows and hands (especially of the thumb) with a score of 6/9 at the Beighton scale, hockey-stick palm crease on her left hand, bilateral fifth finger clinodactyly, one hyperpigmented macule on the abdomen and on the left-knee. Neurological examination resulted normal. Dysmorphic features of the patient included midfacial hypoplasia, bilateral epicanthic 
fold, upslanting palpebral fissures, nasal bone hypoplasia, dental occlusion of Class III (Fig. 1). It was reported that she underwent extractions of two deciduous teeth (inferior right posterior deciduous molars) due to caries; at the time of the last visit, other deciduous teeth (inferior left deciduous canines and molars) were still decayed as well as the residual roots of upper right and left last deciduous molars. The inferior second left deciduous molar was already treated with restoration. Through oral investigation, dental OPT-XR and CT we found dental anomalies of shape, number and position. The clinical crowns of the erupted upper incisors had a diameter smaller than usual; moreover, they were cylindrical, with a horny look and presented with a depression in the middle of the incisal portion (peg- shaped and notched). The dental enamel was affected by areas of demineralization (white and yellow-brown spots), the left central inferior permanent incisor had a lack of enamel (pitting) on the vestibular surface. White and brown spots were diffuse (Fig. 2a). Dental agenesis of the two upper canines was found; the germs of the wisdom teeth were not visible. The first right premolar had a dystopic eruptive direction (distoangulation), and it was still in inclusion (Fig. 2b).

Patient 2, the younger sister of Patient 1, was referred for genetic counselling at five years of age, due to a cardiac disease and positive family history for $1 \mathrm{p} 36$ deletion syndrome. The baby was born by cesarean section at $39^{+6} \mathrm{GW}$ because of breech presentation. Birth weight was $3120 \mathrm{~g}$ (10-50th centile) [12], birth length was 48 $\mathrm{cm}$ (10-50th centile) [12] and OFC was $36.2 \mathrm{~cm}$ (>97th centile) [12]. Newborn hearing screening was normal. Immediately after birth, in order to investigate a systolic murmur, an echocardiogram was performed revealing a patent arterial duct and patent foramen ovale, slight flattening of the interventricular septum, mild tricuspid deficiency and dilated cardiomyopathy with left-ventricular non-compaction. At five months of age, she developed left heart failure (Ejection Fraction $=23 \%$ ) and started a

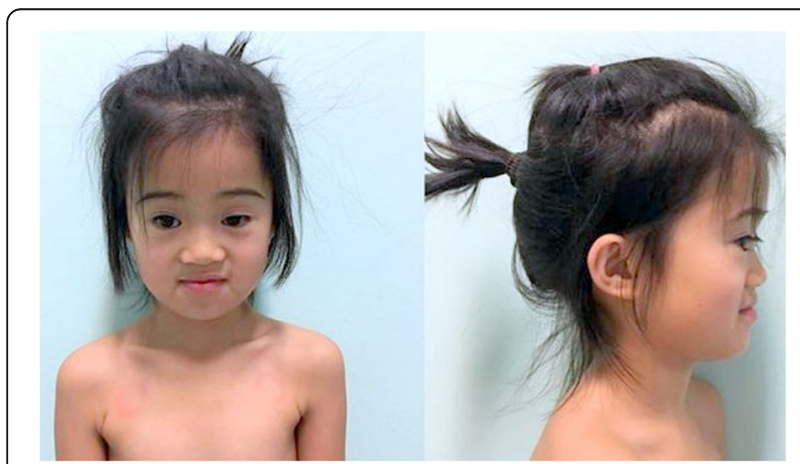

Fig. 1 Patient 1. Dysmorphic features: midfacial hypoplasia, bilateral epicanthic fold, upslanting palpebral fissures, palpebral ptosis, nasal bone hypoplasia, dental occlusion of Class III

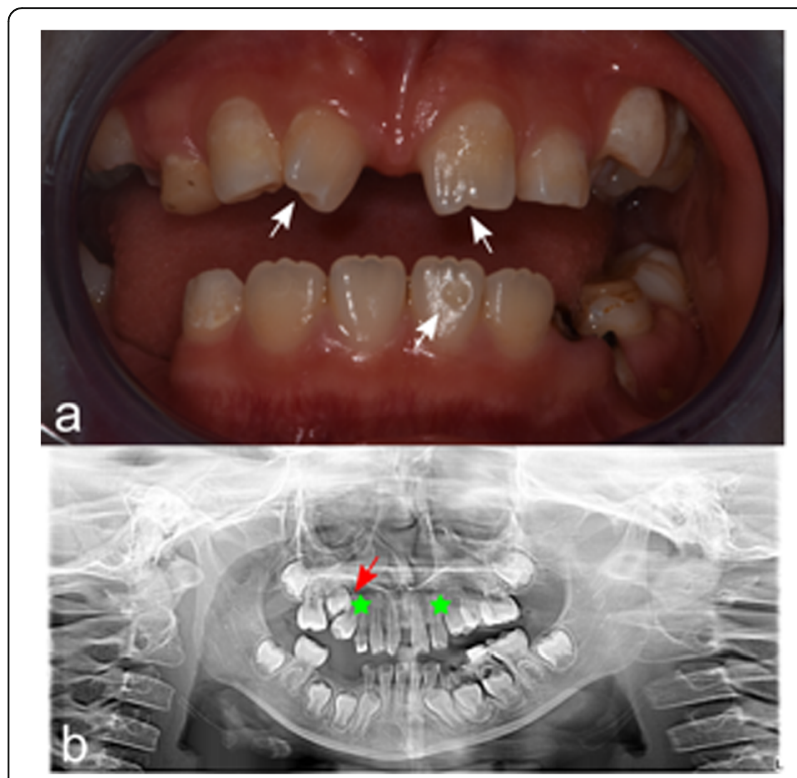

Fig. 2 Patient 1. a Dental anomalies. Note the atypical shape of the permanent upper incisors (peg- shaped and notched; white arrows) and the areas of enamel demineralization (white and yellow-brown spots diffused and the lack of enamel -pitting-) on the vestibular surface of the inferior central incisor (white arrow). b Orthopantomography X-Ray (OPT-XR). The dental agenesis of the two upper canines was identified (canine position is indicated with stars) together with the dystopic eruptive direction (distoangulation) of the still included upper second premolar (red arrow). The germs of the wisdom teeth were not visible

treatment with metoprolol, digoxin, furosemide, captopril and warfarin. Two months later, she suffered from right frontal subdural hemorrhage with epileptic seizure and she was treated with midazolam and surgical drainage. A history of poor growth due to feeding difficulties, that required a nasogastric tube, and a moderate hypotonia are documented in her medical records. Developmental milestones were almost normal: she walked at 13 months of age and spoke at 12 months. Despite this, since the age of 4 she has been undergoing speech therapy rehabilitation for a mild language delay and she is making gradual progress. She speaks both Italian and Chinese. At 6-year-old she was on treatment with metoprolol, ramipril, levetiracetam and acetylsalicylic acid. Electroencephalograms continue to show epileptogenic anomalies. Genetic analysis (SNP array) identified the same 1 p36.32 deletion of the sister. SNP-array does not identify any anomalies in the parents, suggesting a germline mosaicism. Paternity was confirmed. Intellectual developmental evaluation at 6-year-old with the WPPSI-III showed a total IQ of 78. The Otorhinolaryngologist consult indicated a normal bilateral hearing. Eye examination showed strabismus, therefore corrective lenses were prescribed and later surgery was performed. At the time of the last visit (6 years), her height was $107 \mathrm{~cm}$ (3-10 centile) and weight was $16 \mathrm{~kg}$ ( $3-10^{\circ}$ centile). 
Physical exam was significant for post-natal microcephaly, ligamentous laxity of the elbows with a score of $2 / 9$ at the Beighton scale. Hockey-stick palm crease on her right hand, bilateral short fifth finger and one hyperpigmented macule on the back were also observed. Dysmorphic features of the patient included bilateral epicanthus, low-set ears, severe midfacial hypoplasia, flat occiput, two posterior hair whorls, high palate (Fig. 3). Neurological exam resulted normal. The odontostomatological examination revealed a severe III Class malocclusion in a micrognathia pattern with a right posterior crossbite, massive caries of the deciduous upper incisors, small caries of both second upper deciduous molars. The first deciduous molars have been already treated with restoration. Areas of demineralization (white and brown spots) are diffused (Fig. 4a). X-Ray and CT examination showed agenesis of the upper canines and malposition of the central right upper incisor and the lateral left upper incisor; following the X-ray report, a shape anomaly of the upper incisors is suspected, though it needs to be confirmed after the eruption (Fig. 4b).

SNP-array analysis (ILLUMINA ExpressExome arrays) on blood samples of the two sisters identified a deletion $1 \mathrm{p} 36.32$ of about $2.3 \mathrm{Mb}$ (arr [hg19] 1q36.32(2.309.082 $\times$ $2,2.310 .193-4.634 .978 \times 1,4.636 .767 \times 2)$ ), typical of the 1 p36 deletion syndrome (Fig. 5). This analysis was performed also on the parents, demonstrating that they did not carry any pathogenic microduplication or microdeletion and confirming the paternity. Genotype data of the SNPs located in the deleted region allowed to prove that the 1 p36.32 deletion occurred on the maternal allele. Karyotype analysis and Fluorescent In Situ Hybridization analysis (FISH analysis) with probes Vysis LSI P58 and Vysis 1pTEL did not showed subtelomeric rearrangements or chromosomal translocations in the parents (see Additional file 1). Clinical exome sequencing (Clinical Exome Solution-SOPHiA Genetics) was performed on the patient 1 to exclude small deletions/duplications or

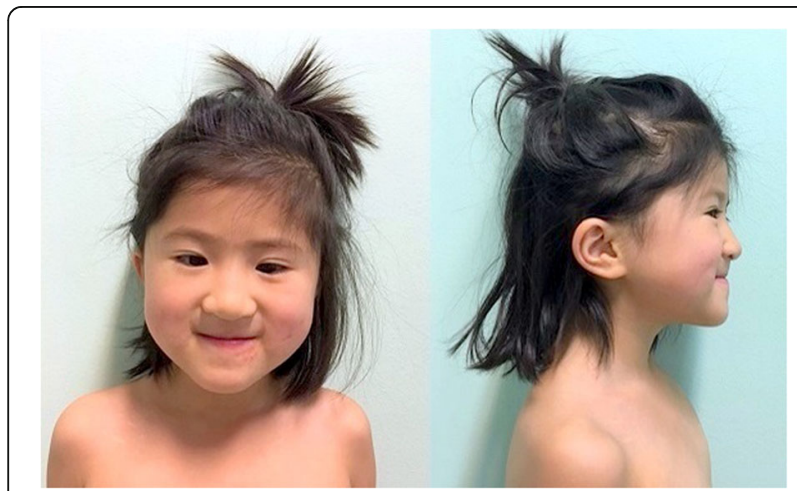

Fig. 3 Patient 2. Dysmorphic features: bilateral epicanthus, low-set ears, severe midfacial hypoplasia, flat occiput

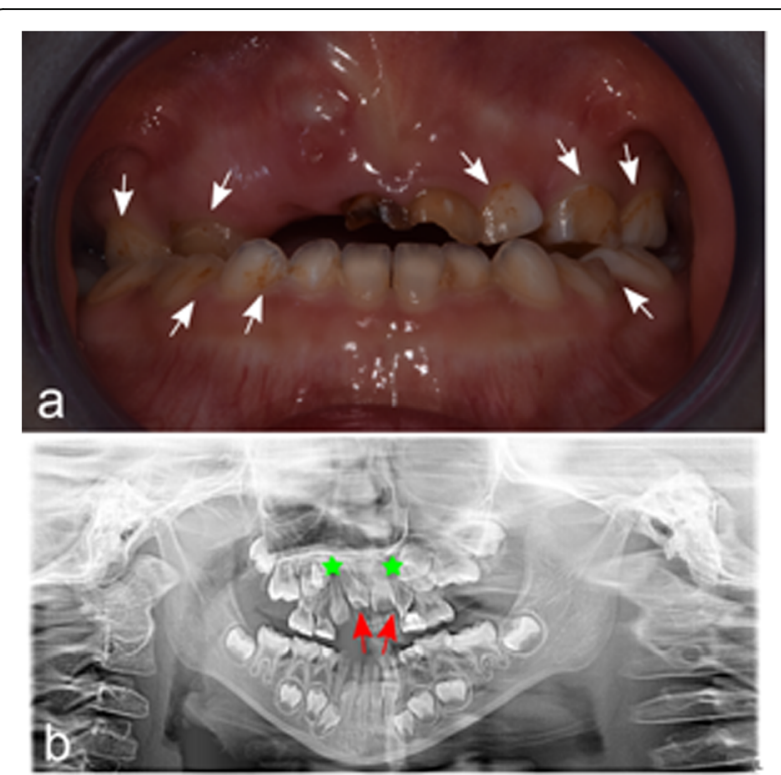

Fig. 4 Patient 2. a Dental anomalies. Note the diffused demineralization (white and brown spots; white arrows). b Orthopantomography X-Ray (OPT-XR). Dental agenesis of the two upper canines was identified (canine position is indicated with stars). The suspected shape anomaly of the upper incisors is indicated with red arrows

single nucleotide variants in known genes associating with dental anomalies.

During the evaluation of the patients, the mother informed us she was pregnant and at gestational age of 12 weeks she underwent prenatal Comparative Genomic Hybridization-array (CGH-array) analysis (DNA extracted from chorionic villous sampling). CGH-array was performed by a different laboratory and revealed that the fetus did not carry the 1 p36 deletion or other quantitative alterations.

\section{Discussion and conclusions}

To date, no dental anomalies in patients with 1p36 deletion syndrome have been reported in literature. Our patients, however, presented with an uncommon clinical feature, namely the dental anomalies. Interestingly, this characteristic was the only phenotypic feature shared by both sisters. The oral phenotype is well represented and characterized by malocclusion, canine agenesis and dental malposition. The shape anomaly found in the permanent upper incisor is uncommon and it was similar to the Hutchinson's incisors sign of congenital syphilis. Only after their eruption, it will be possible to confirm whether the permanent teeth of the younger sister show the same shape and structural dental anomalies recorded for the oldest sister. Both patients had a severe III Class occlusion, while both their parents had a normal occlusion of Class I. Tooth agenesis is the most common 


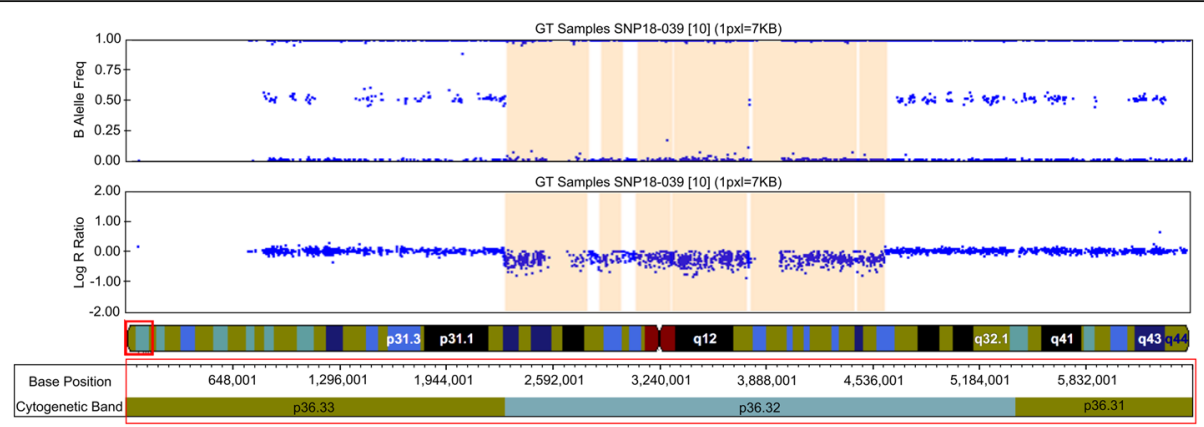

Fig. 5 Oligonucleotides array profile of our patients shows 1p36.32 deletion of 2,3 Mb. OMIM genes disease-causing inside the critical region 1 p36.32, according UCSC: PEX10, PRDM16, CEP104

craniofacial malformation in humans [13]. It should be reported that the mother presented the agenesis of the left upper lateral incisor, the most frequent dental agenesis of permanent teeth with the mandibular second premolars $[14,15]$, while the maxillary canines were missing in our syndromic patients.

Both of the children had a high cario-receptivity despite a good oral hygiene; however, it is not possible to establish whether this is due to the structural anomaly in the deciduous teeth or to sugar-rich diet. The surface with yellow-brown spots of the permanent teeth of patient 1 suggested a diffuse hypomineralization with an increased caries risk.

In the light of the present findings, an early dental examination and an orthodontic visit with a strictly follow up are highly recommended in order to avoid the insurgence of caries. Moreover, an interceptive orthodontic therapy for an initial III Class correction would minimize the future problem of dental agenesis and malposition disturbances in the spacing of the dentition and would allow an adequate nutrition for an appropriate growth and development. Furthermore, even though they are easily recognizable with a simple oral investigation, so many anomalies of shape, number, position and enamel surface associated with a severe III Class are rarely found in clinical practice. Therefore, they could be the cue for further investigation of several organs and genetic analysis.

We searched for OMIM genes included in the 1p36 deletion and we did not find candidate genes that may cause impaired odontogenesis. To exclude the co-segregation of $1 \mathrm{p} 36$ deletion with pathogenic variant/s in known genes associating with dental anomalies, we performed clinical exome sequencing on the patient 1 . No small deletions/ duplications or point mutations were identified in candidate genes (see Additional file 2). Hence, even though we cannot attribute this aspect unquestionably to monosomy $1 \mathrm{p} 36$, it is likely that this is an underdiagnosed finding of 1 p36 deletion syndrome spectrum.

To date, there are two reports of a recurrent monosomy $1 \mathrm{p} 36$ due to probable germline mosaicism. Our data, as well as the findings of Di Donato $\mathrm{N}$ et al. (2014) and Gajecka M et al. (2010), confirm that the germline mosaicism seems to be the only explanation for the presence of the same $1 \mathrm{p} 36$ deletion in two sisters born from healthy parents $[2,5]$.

The normal result of CGH-array of the fetus supports the hypothesis of a germline mosaicism. Our findings, as well as Di Donato $\mathrm{N}$ et al. (2014), confirm that the recurrence risk for a couple that has a child with a de novo 1p36 deletion might be higher than the general population risk [2] and that germline mosaicisms may be more common than expected.

Our patients present with a very peculiar phenotype. Patient 1 has no cardiac malformations, seizures or language delay, while patient 2 has dilated cardiomyopathy with left-ventricular non-compaction, epilepsy, strabismus, history of poor growth, hypotonia and mild language delay.

Although Patient 1 was referred for suspected learning disorder, she has an IQ of 75 and she attends the primary school full time without any support. The second patient has an IQ of 78 and presents a mild psychomotor delay. It is also significant to consider that the girls speak three languages, Chinese at home and Italian and English in school. We suggest that most of the patients with 1 p36 deletion syndrome have been described with severe intellectual disability for selection bias as arrays analysis is performed more often in patients with lower IQs and with severely impaired cognitive function.

Di Donato at al [2] described two brothers with 1 p36 deletion syndrome not involving KCNAB2 and GABRD genes and without epileptic history. They, therefore, supposed that haploinsufficiency of one of these genes could cause epilepsy in patients with 1 p36 deletion syndrome. However, the deletion found in our probands does not involve KCNAB2 and GABRD genes or other OMIM genes known to be related to epilepsy, but nevertheless patient 2 has an epileptic history. Therefore, we suggest that haploinsufficiency of genes other than KCNAB2 and $G A B R D$ may cause seizures in patients with 1 p36 deletion with an incomplete penetrance. 
The deletion of our patients involves the PRDM16 gene, previously associated with cardiomyopathy in $1 \mathrm{p} 36$ deletion syndrome [16]. The fact that only one of our patients suffers from cardiomyopathy supports the hypothesis that the penetrance of PRDM16 haploinsufficiency is incomplete and thus the deletion of this gene is not enough to develop cardiomyopathy, as previously reported [2].

In conclusion, we report the third study with recurrent $1 \mathrm{p} 36$ deletion syndrome in two siblings, due to a germline mosaicism. Our two patients show very mild intellectual impairment and present with dental anomalies, never described before in association with 1 p36 deletion syndrome. This report suggests that 1 p36 deletion syndrome may presents also with mild cognitive impairment or even with normal intellectual development, as previously described [2]. This finding is very important for genetic counselling, especially in a prenatal setting. We suggest that $1 \mathrm{p} 36$ deletion syndrome may be sometimes underdiagnosed because of the possible absence of cognitive phenotype impairment. Furthermore, we believe that the dental anomalies should be investigated in 1 p36 deletion syndrome and that the spectrum of the condition could be broader than we assume.

\section{Supplementary information}

Supplementary information accompanies this paper at https://doi.org/10. 1186/s12887-020-02049-1.

Additional file 1: Figure $\mathbf{6}$ - FISH analysis. FISH analysis reveals the presence of 1 p36 region in each chromosome 1 . The arrow indicates 1 p36 region, the orange spot corresponds to CDK11B gene and the green spot corresponds to AGRN gene. The dotted arrow indicates 1q25 region to demonstrate that this is the chromosome 1. a) Father assay; b) Mother assay; c) Probes used for the analysis: LSI P58 (orange) and Vysis $1 \mathrm{pTeL}$ (green) for 1 p36 region; LSI 1q25 (aqua) for 1q25 region.

Additional file 2. Clinical Exome Sequencing (SOPHiA) of the patient 1. Table summarizes genes analysed and the coverage for each exon. No pathogenic/likely pathogenic variants were identified in candidate genes (WNT10A, MSX1, LRP6, WNT10B, PAX9, EDA, AXIN2, EDARADD, FGFR1, IRF6) or in genes included in the virtual panel generated by SOPHiA Software (SOPHiA-DDM-v4) using the following HPO terms: oligodontia; dental anomalies (virtual panel: ANKRD11; AXIN2; BCOR; CCBE1; CLDN1; EDA; EDARADD; FAT4; KCNJ5; POLR3A; RNU4ATAC; FGFR1; HOXD13; IRF6; KCNJ2; LRP6; LTBP3; MSX1; NAA10; PAX9; POLR1C; POLR3B; PORCN; PTHLH; SATB2; SH3BP2; SLC29A3; TGFA; TP63; WNT10A).

\section{Abbreviations}

OFC: Occipito Frontal Circumference; CT: Computed Tomography; MRI: Magnetic Resonance Imaging; FISH: Fluorescent In Situ Hybridization; SNP-array: Single Nucleotide Polymorphism-array; OPT-

XR: Orthopantomography X-Ray; IQ: Intelligence Quotient; WPPSI-III: Wechsler Preschool and Primary Scale of Intelligence, Third Edition; $\mathrm{CGH}$ array: Comparative Genomic Hybridization-array

\section{Acknowledgements}

The authors would like to thank Mr. Michele Mafrici for review of English language of the manuscript.

\section{Authors' contributions}

ND, BM and BE analyzed and interpreted clinical features of patients. GF, SF and GP analyzed and interpreted clinical features of patients and the results of genetic analysis. DAAP and PB performed SNP-arrays, FISH and karyotype.
NCO and CM analyzed and interpreted dental anomalies. MA performed the last follow-up visits and interpreted the Clinical Exome Sequencing. GE performed Clinical Exome Sequencing and revised the manuscript. All authors read and approved the final manuscript.

\section{Funding}

No funding was received for this case report

Availability of data and materials

All data generated or analysed during this study are included in this published article.

Ethics approval and consent to participate

All procedures performed are in accordance with the ethical standards.

\section{Consent for publication}

Written consent for publication was obtained from the parents of the patients.

\section{Competing interests}

The authors declare that they have no competing interests.

\section{Author details}

${ }^{1}$ University of Trieste, Trieste, Italy. ${ }^{2}$ University of Padua, Padua, Italy. ${ }^{3}$ Institute for Maternal and Child Health IRCCS Burlo Garofolo, Trieste, Italy.

${ }^{4}$ Department Medical Sciences, University of Torino, Torino, Italy. ${ }^{5}$ Division of Medical Genetics, AAS n.5 Friuli Occidentale, Pordenone, Italy.

Received: 26 August 2019 Accepted: 25 March 2020

Published online: 09 May 2020

\section{References}

1. Jordan VK, Zaveri HP, Scott DA. 1 p36 deletion syndrome: an update. Appl Clin Genet. 2015;8:189-200.

2. Di Donato N, Klink B, Hahn G, et al. Interstitial deletion 1 p36.32 in two brothers with a distinct phenotype e overgrowth, macrocephaly and nearly normal intellectual function. Eur J Med Genet. 2014;57(9):494-7.

3. Shimada S, et al. Microarray analysis of 50 patients reveals the critical chromosomal regions responsible for $1 \mathrm{p} 36$ deletion syndrome-related complications. Brain and Development. 2015;37(5):515-26.

4. Battaglia A. 1 p36 Deletion Syndrome. 2008 Feb 1 [updated 2013 Jun 6]. In: Adam MP, Ardinger $\mathrm{HH}$, Pagon RA, et al., editors. GeneReviews ${ }^{\circledast}$ [internet]. Seattle (WA): University of Washington, Seattle; 1993-2019. Available from: https://www.ncbi.nlm.nih.gov/books/NBK1191/.

5. Gajecka M, Saitta SC, Gentles AJ, et al. Recurrent interstitial 1p36 deletions: evidence for germline mosaicism and complex rearrangement breakpoints. Am J med genet A. 2010;152A(12):3074-83.

6. Guterman S, Beneteau C, Redon S. Prenatal findings in 1 p36 deletion syndrome: new cases and a literature review. Prenat Diagn. 2019. https:// doi.org/10.1002/pd.5498.

7. Carter LB, Battaglia A, Cherry A. Perinatal distress in 1p36 deletion syndrome can mimic hypoxic ischemic encephalopathy. Am J Med Genet A. 2019. https://doi.org/10.1002/ajmg.a.61266.

8. Nobili V, Mosca A, Francalanci $P$, et al. First case of nonalcoholic steatohepatitis in a child with del (1p36) and dup (Xp22): review of the literature. Clin Dysmorphol. 2018;27(2):42-5.

9. Zhang Z, Wang J, Li N, et al. Cutis laxa in a patient with 1 p36 deletion syndrome. J Dermatol. 2018;45(7):871-3.

10. Chawla $V$, Anagnost MR, Eldemerdash AE, et al. A novel case of biliary atresia in a premature neonate with 1p36 deletion syndrome. J Investig Med High Impact Case Rep. 2018;24(6):2324709618790613. https://doi.org/ 10.1177/2324709618790613 eCollection 2018 Jan-Dec.

11. American Psychiatric Association. Diagnostic and Statistical Manual of Mental Disorders (DSM-5). 5th ed. Washington, DC: American Psychiatric Association; 2013. p. 13-37.

12. Villar J, et al. International standards for newborn weight, length, and head circumference by gestational age and sex: the Newborn Cross-Sectional Study of the INTERGROWTH-21st Project. Lancet. 2014;384(9946):857-68.

13. Matalova E, Fleischmannova J, Sharpe PT, et al. Tooth agenesis: from molecular genetics to molecular dentistry. J Dent Res. 2008;87(7):617-23. 
14. Polder BJ, Van't Hof MA, Van der Linden FP, et al. A meta-analysis of the prevalence of dental agenesis of permanent teeth. Community Dent Oral Epidemiol. 2004;32(3):217-26.

15. Symons AL, Stritzel F, Stamation J. Anomalies associated with hypodontia of the permanent lateral incisor and second premolar. J Clin Pediatr Dent. 1993;17(2):109-11.

16. Arndt, et al. Fine mapping of the 1 p36 deletion syndrome identifies mutation of PRDM16 as a cause of cardiomyopathy. Am J Hum Genet. 2013;93(1):67-77.

\section{Publisher's Note}

Springer Nature remains neutral with regard to jurisdictional claims in published maps and institutional affiliations.

Ready to submit your research? Choose BMC and benefit from:

- fast, convenient online submission

- thorough peer review by experienced researchers in your field

- rapid publication on acceptance

- support for research data, including large and complex data types

- gold Open Access which fosters wider collaboration and increased citations

- maximum visibility for your research: over $100 \mathrm{M}$ website views per year

At $B M C$, research is always in progress.

Learn more biomedcentral.com/submissions 